\title{
Unmasking the standing wave of invisibility: An account in terms of object-mediated representational updating
}

\author{
Elisabeth Hein and Cathleen M. Moore \\ University of Iowa, Iowa City, Iowa
}

\begin{abstract}
A central bar presented in counterphase with two flanking bars creates the perception of only two bars, instead of three, flickering (standing wave of invisibility illusion). Current explanations of this illusion highlight the importance of local interactions between the central bar and the flankers as a reason for the invisibility of the central bar. In three experiments, we show that the reduction in visibility of the central bar occurs even when the flankers are spatially separated from the central bar. Thus local mechanisms-low-level lateral inhibition or border-ownership competition - do not suffice to account for the decreased visibility. Furthermore, the reduced visibility of the central bar is accompanied by the perception of the flankers in apparent motion at all separations. We suggest an account of the standing wave phenomenon in terms of object updating: The representation of the central bar is updated with the representation of the flankers leaving the perception of just the flankers moving across space. The stimuli for the key conditions from this study, in QuickTime format, may be down loaded from http://app.psychonomic-journals.org/content/supplemental.
\end{abstract}

Lateral masking is a specific form of masking in which an otherwise visible stimulus can be rendered invisible by another stimulus that either precedes (paracontrast masking) or follows (metacontrast masking) that stimulus. This masking effect occurs even though the two stimuli never spatially or temporally overlap (see Breitmeyer \& Öğmen, 2000, and Weisstein, 1972, for a review). Similarly, when a central stimulus is presented flickering in counterphase with an adjacent flanking stimulus in a continuous cycle, the central stimulus becomes invisible, leaving the perception of only the periodically flickering flanking stimuli. This phenomenon was first observed by Werner (1935), who reported a series of experiments using a variety of alternating stimuli-for example, disc and ring stimuli and bar stimuli (see Figure 1). It has more recently been referred to as the standing wave of invisibility illusion (Macknik \& Livingstone, 1998). Werner found that visibility depended critically on the interval between the central and the flanking stimulus, as well as on the time between series of these stimuli. Unlike classic metacontrast and paracontrast masking, the continuous cycling of the stimuli in the two frames gives rise to a relatively stable visual percept that can be considered by the observer over a protracted period of time. This feature makes the standing wave illusion a useful tool for studying the formation and updating of visual representations over time.

It remains unclear what controls the visibility of the central stimulus in the standing wave illusion. Theories of masking in general explain lateral masking in terms of in- hibitory mechanisms in early levels of visual processing, whereby the representation of one stimulus is interrupted and replaced by the representation of the other stimulus (e.g., Breitmeyer \& Ganz, 1976; Francis, 1997; for a review, see Breitmeyer \& Öğmen, 2006). Following this line of theories, Macknik and Livingstone (1998) offered an explanation of the standing wave illusion in terms of lateral inhibitory processes. They did single-unit recordings in early visual areas (V1 and LGN) and presented a central bar cycling continuously with two flanking bars to awake rhesus monkeys. The central bar was presented in the neurons' receptive fields and was oriented to match the preferred orientation of a given neuron. Macknik and Livingstone measured responses of neurons to the central bar both when it was presented in counter phase with the flanking bars and when it was presented alone. Neuronal responses to the central bar were inhibited in the flanked condition relative to the central bar alone condition, a pattern that parallels the phenomenology. On the basis of these and related results, Macknik and colleagues (see Macknik \& Martinez-Conde, 2007, for a review) proposed that the standing wave illusion is caused by the interaction of the spatiotemporal edges of central bar and flanking bars in a lateral inhibitory network. In their view, the flanking bars provide a combination of meta- and paracontrast masks that causes the central bar to disappear.

Werner (1935) also explained the standing wave phenomenon in terms of lateral interactions. However, he hypothesized that the interactions were at the level of 
Presentation

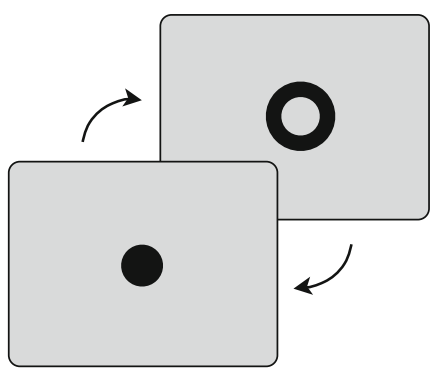

Presentation

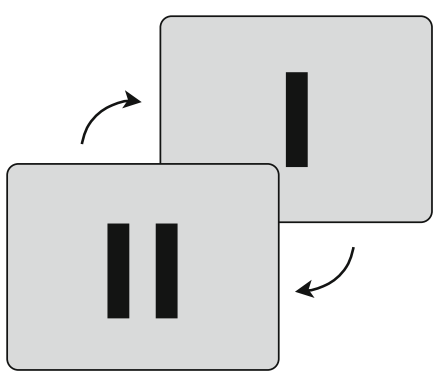

Perception

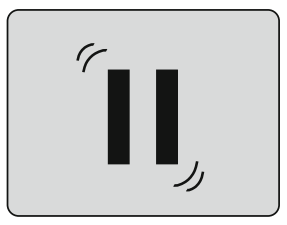

Figure 1. The standing wave illusion with the disc and bar stimuli used in different studies. Werner (1935) mainly used disc and ring stimuli, illustrated in the upper panel of the figure, whereas in more recent studies (e.g., Enns, 2002; Macknik \& Livingstone, 1998), as well as in the present study, bar stimuli were used, similar to those illustrated in the lower panel of the figure.

contour formation. Werner assumed that the formation of a stimulus representation starts with the contour and constructs its surface from that contour as a second step. If the process of forming a representation of the central stimulus is interrupted by the flanking stimulus before a stable representation can be established (i.e., before the second step of surface construction takes place), the representation of the central stimulus will be lost. The already established representation of the contour, however, will be used instead to construct the representation of the flanking stimulus. In a series of experiments, Werner examined the influence of shape similarity on the visibility of the central stimulus and found that the more dissimilar the two sets of stimuli were, the less masking was observed. From this, he concluded that contour confusion is greatest when contours are similar.

Enns (2002) offered a similar account to that of Werner (1935). He proposed that the shared border between the central and flanking stimuli leads to a competition for border ownership and, by extension, for object representation. The central stimulus - a bar - is at a disadvantage, because both of its boundaries are shared, whereas the flanking stimuli - two bars - each have one border that is undisputed. This advantage results in the flankers being represented and perceived over the central bar. Consistent with this hypothesis, when the flankers were themselves flanked by yet another set of bars that was presented in phase with the central bar (Figure 2), the central bar be- came visible again (Enns, 2002). In this case, the immediately flanking bars have two disputed boundaries, just like the central bar. The central bar, however, becomes grouped with the outer flanking bars by virtue of appearing in the same frame as they do. Because the outer bars have undisputed boundaries, they win the competition for representation and pull the central bar along with them into visibility. This finding renders any account in terms of local lateral inhibition incomplete; the local edge interactions are the same as in the original conditions, yet the central bar is visible in one case (flanked flankers) and not in the other (original conditions).

Although explanations in terms of border competition emphasize the importance of factors other than just the spatiotemporal edges of the stimuli, they nonetheless depend on the proximity of borders for the interference to occur. In this sense, they are similar to local lateralinhibition accounts.

A more recent class of masking theories emphasizes interactions between object representations and, therefore, does not depend necessarily on proximity (Duangudom, Francis, \& Herzog, 2007; Enns \& Di Lollo, 1997). These theories are supported in part by studies showing that the influence of the global configuration of the masks can be as great as or greater than that of their local contours and overall luminance strength (e.g., Herzog, Fahle, \& Koch, 2001). In line with this idea, Werner (1935) showed that when the central disc in one of his cycling displays was shown as one element among a circle of rings, it suffered little or no masking. This was true despite the fact that the local configuration produced nearly perfect masking when the disc was presented alone. This is similar to Enns's (2002) finding that flanking the flankers with additional bars eliminated the standing wave illusion.

In view of these findings, we propose an interpretation of the standing wave in terms of object updating to account for the object-level effects of the standing wave. This account relies on the assumption that representational updating is mediated through object representations (Enns, Lleras, \& Moore, 2008; Lleras \& Moore, 2003; Moore \& Lleras, 2005; Moore, Mordkoff, \& Enns, 2007). Given the right timing between the central bar and flankers, the visual

Presentation

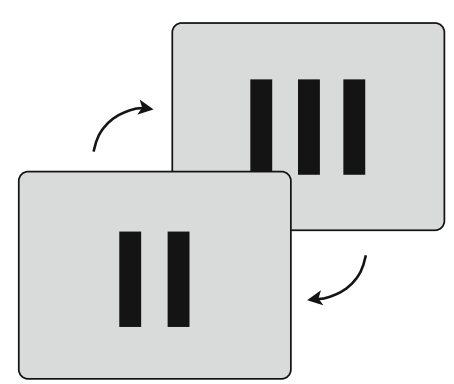

Perception

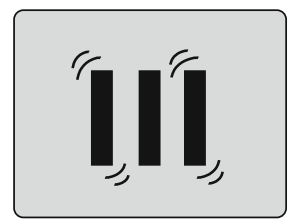

Figure 2. The modified standing wave illusion, in which a second set of flankers is placed adjacent to the original flankers at the same time as the central bar is presented. The central bar is visible in this case. 
system interprets them not as separate objects, but as one object at different locations. Consequently, only one representation is constructed and is updated over time, modifying the original representation by integrating new and old information about the stimulus. If, on the other hand, the timing is not appropriate for an interpretation of the two sets of objects as one, separate object representations are formed, the representation of the central bar is not updated, and therefore, the central bar becomes visible.

According to the object substitution framework, the more similar the central and flanking stimuli are, the more likely it is that the representation of the central stimulus will be updated by the representation of the flanking stimuli. Consistent with this, it has been found that shape and surface similarity seem to influence the visibility of the central stimulus in the standing wave display (Enns, 2002; Pilling \& Gellatly, 2009; Werner, 1935). In addition, if the scene is interpreted as the central bar being a previous instantiation of the flanking bars, it is expected that apparent motion should be seen between them (Lleras \& Moore, 2003). Taken together, the more similar the central bar and flankers are, the more likely they are to be integrated, and therefore observers should perceive more motion. This interpretation is supported by results from Pilling and Gellatly, who found that participants reported perceiving stronger apparent motion when the shape of the central bar and the flankers were identical than when they were different.

In this study, we focused on the importance of the shared or very nearby boundaries of the central and flanking bars in the standing wave. Whereas local mechanisms, such as those in the border-ownership and low-level inhibitory accounts, depend on close proximity between stimulus borders, explanations in terms of object-mediated influences do not. In two experiments, we manipulated the spatial separation between the central bar and the flanking bars, as well as the duration of the flanking stimuli, in order to manipulate the visibility of the central bar. Both the low-level inhibitory and border-ownership accounts predict that the visibility of the central bar should increase dramatically with increasing separation. Low-level inhibitory accounts predict this result because they are explicitly concerned with spatial proximity. Border-ownership accounts predict this result because the central bar should be invisible only when the boundaries between central bar and flankers are in dispute. This will be when they are very near each other or overlapping. In contrast, the object-updating hypothesis predicts that the central bar will be rendered invisible across a much larger range of separations. This result would follow because updating is expected to occur under the same conditions as apparent motion, which of course occurs across a broad range of spatial separations (e.g., Korte, 1915). To examine the relationship between visibility and apparent motion, in the third experiment we used the same conditions as in the first but collected judgments of motion quality instead of judgments of central-bar visibility. The results show that central-bar visibility did depend on separation, suggesting that local mechanisms do play a role. However, central- bar visibility was still poor across even large separations. Thus, local mechanisms cannot account for all of the decreased visibility. Moreover, those conditions that yielded lower central-bar visibility also yielded higher ratings of good apparent motion. We propose that an object updating process, which also plays a role in apparent motion, contributes significantly to the standing wave phenomenon.

\section{GENERAL METHOD}

\section{Participants}

Different sets of 16 undergraduate students from the University of Iowa participated in each of the three experiments. They were naive to the purpose of the experiment and received course credit in exchange for participating in the experiment. None participated in more than one experiment. All of them reported normal or correctedto-normal visual acuity and normal color vision. Two participants in Experiment 2 had to be replaced, because they did not finish the experiment within the available time $(1 \mathrm{~h})$.

\section{Apparatus}

The experiment was controlled by a Macintosh computer (Mac OS X, Version 10.4.10) driving a 17 -in. color monitor with a spatial resolution of $1,024 \times 768$ pixels and a refresh rate of $100 \mathrm{~Hz}$. Stimuli were presented on the screen using MATLAB software (Version 7.4 release 2007a, MathWorks, Natick, MA) with the Psychophysics Toolbox extensions (Version 3.0.8, flavor beta; Brainard, 1997; Pelli, 1997). The participants sat in front of the computer monitor at a distance of about $60 \mathrm{~cm}$.

\section{Stimuli}

Our stimuli were very similar to the ones used by Enns (2002). Two arrays, each consisting of three individual bars, were presented on a middle-gray background $\left(40.1 \mathrm{~cd} / \mathrm{m}^{2}\right)$. All of the bars were black $\left(0.5 \mathrm{~cd} / \mathrm{m}^{2}\right), 3.0^{\circ}$ high and $0.6^{\circ}$ wide. Following Enns, we included two arrays in order to encourage the observers to remain fixated. The arrays were identical except for position. The observers could base their judgments on either or both of them. Each standing wave display was presented $1.3^{\circ}$ of visual angle above and below a black central fixation cross $\left(0.4^{\circ}\right)$. To prevent the two displays from forming a line with fixation, we centered the upper array $0.3^{\circ}$ to the right of center and the lower display $0.3^{\circ}$ to the left of center. In Experiments 1 and 3, the central bars of each array always stayed at the same position, and the flanking bars were presented according to the seven different separations: directly next to the central bar or $0.06^{\circ}, 0.12^{\circ}, 0.25^{\circ}, 0.50^{\circ}, 0.99^{\circ}$, or $1.98^{\circ}(2,4,8,16,32$, or 64 pixels, respectively) away from the central bar. In Experiment 2, each standing wave display was presented $3.0^{\circ}$ to the left and right of a black central fixation cross $\left(0.4^{\circ}\right.$ high and wide). This time, the flanking bars were always presented at the same position, and the central bar was presented directly between the flankers or displaced vertically above or below the flankers. The central bar on the left side was always presented above the flankers, whereas the central bar on the right side was presented below the flankers (see Figure 3 for an illustration of the differences between Experiments 1 and 2). The vertical separation was directly above the flankers, which we will refer to as $0^{\circ}$ shift $; 0.06^{\circ} ; 0.12^{\circ} ; 0.50^{\circ} ; 0.99^{\circ}$; or $1.98^{\circ}(0,2,4,16,32$, or 64 pixels, respectively) away from the upper (or lower) border of the smaller side of the flankers.

\section{Task}

The task in Experiments 1 and 2 was to judge the visibility of the central bar using one of seven possible keys (" 1 " to " 7 " on the central keyboard). The participants were instructed to press the "1" key when the central bar was invisible, the "7" key whenever the central bar was entirely visible; and any key in between, according to how visible 

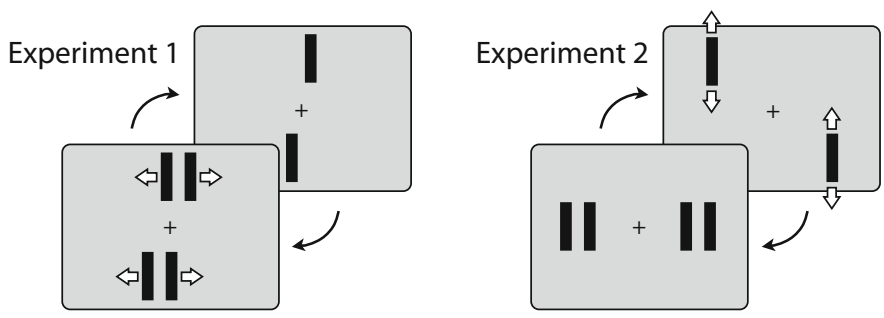

Figure 3. Schematic summary of the differences in the arrangement of the standing wave displays in Experiments 1 and 2. The small white arrows indicate the directions in which the corresponding bars were moved in order to separate central bar and the flankers spatially. In Experiment 1, the central bar and the flankers were presented above and below fixation, slightly shifted to the left and right. In Experiment 2, the flankers were presented to the left and right of fixation, and the central bars were displaced below and above the flankers.

the central bar appeared to them. In Experiment 3, the participants judged the quality of motion between the central bar and flankers on a scale of $1-7(1=$ perfect motion; $7=$ no motion $)$. The participants were encouraged to use the entire scale when responding.

\section{Design}

A 7 (separation) $\times 6$ (flanker duration) within-subjects design was used. Both factors were mixed within blocks of trials. Each observer participated in one experimental session of eight blocks, each consisting of 42 trials. Overall, we collected eight observations for each separation and flanker duration condition for each participant in each experiment.

\section{Procedure}

Written instructions describing the task were presented on the monitor at the beginning of the experiment. After reading the instructions, the observers were shown two extreme examples of the standing wave array at a separation of $0^{\circ}$, where the central bar was usually seen as either invisible (long flanker duration) or very visible (short flanker duration). The experimenter emphasized the importance of remaining fixated on the central cross and encouraged the participants to refixate before judging the visibility of the central bar if they lost fixation. To help the participants maintain fixation, we presented two identical arrays, one above and one below fixation. The participants were informed that these two arrays were identical. The experimental session lasted about $50 \mathrm{~min}$. Breaks were provided every 21 trials. The participants initiated the next block when they were ready to continue by pressing a button on the keyboard. The observers were strongly encouraged to rest as much as they needed between blocks.

The trial events are illustrated in Figure 4. Each trial began with the presentation of the fixation cross. The participants were instructed to fixate and then to press the space bar in order to initiate the trial. As soon as a key was pressed, the two sets of flanking bars appeared. The flankers were presented for a variable (but in a given trial, fixed) time interval of 20,50, 80,110,170, or $300 \mathrm{msec}$, sepa-

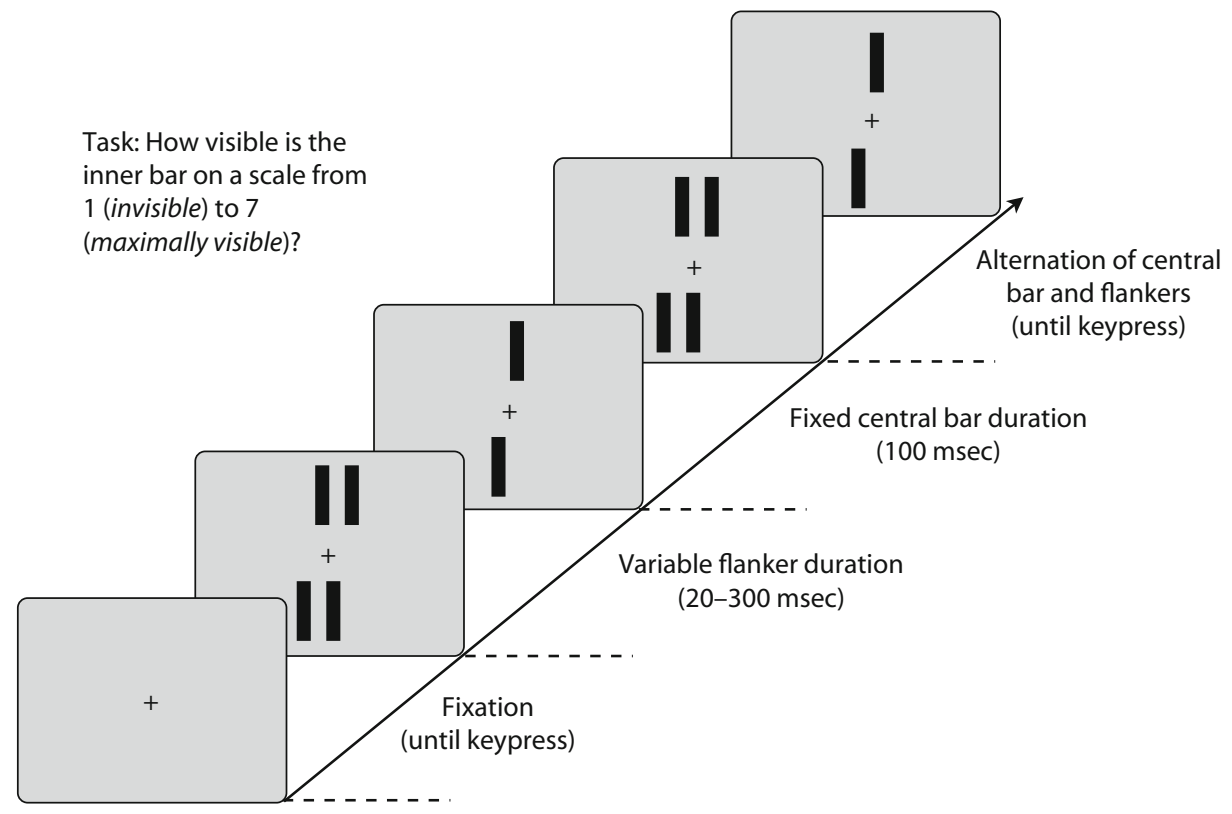

Figure 4. A basic trial sequence in all of the experiments. After the participants initiated the trial, the central bar and flankers were presented in an alternating order until a response key was pressed, indicating how visible the inner bar appeared. This example shows a $0^{\circ}$-separation condition; the illustrated placement of the bars corresponds to those in Experiments 1 and 3. 
rated from the position of the central bar by one of seven different separations. After that, the central bar was presented for $100 \mathrm{msec}$. The flankers and the central bar continued to alternate until the participants responded. The participants were asked to take their time when responding. The standing wave display was presented for at least $1.5 \mathrm{sec}$ before any answer was accepted. To indicate that the response could be given, the fixation cross became thicker. If the participants responded too early or pressed a key other than one of the response keys, a written error message was presented at the center of the display and the trial was aborted. These trials were repeated later during the block. As soon as the participants responded, a blank screen was shown for $500 \mathrm{msec}$ before the fixation cross was presented again and the participants could initiate the next trial.

\section{EXPERIMENT 1}

In Experiment 1, we separated the central bar and the flankers by displacing the flankers horizontally to the left and right of the central bar. Specifically, two identical standing wave displays were presented above and below fixation, slightly displaced to the left and right (see Figure 3, left panel). The flankers were presented at one of seven separations from the central bar, ranging from $0^{\circ}$ to $1.98^{\circ}$. The central bar was presented for $100 \mathrm{msec}$, and flanker duration varied from 20 to $300 \mathrm{msec}$. The task was to indicate on a scale from 1 (invisible) to 7 (very visible) how visible the central bar was.

\section{Results and Discussion}

Figure 5 shows mean visibility ratings as a function of separation and flanker duration in Experiment 1. The $0^{\circ}$-separation condition was essentially a replication of Enns (2002). The observers reported the central bar as highly visible at very short flanker durations $(20 \mathrm{msec})$ and nearly invisible at longer flanker durations (170$300 \mathrm{msec}$ ). Critical to the question being addressed in this study, this general pattern is evident at all of the separations. The main difference is that at the largest separations, the lowest visibility rating was not as low as in the closer conditions, and the variability of responses (note the error bars) was also increased.

Statistical analyses confirmed these apparent patterns. Mean visibility ratings for individual observers were submitted to a 7 (separations) $\times 6$ (flanker durations) ANOVA. Alpha was set at .05 for these and all subsequent analyses. When appropriate, $p$ values were GreenhouseGeisser corrected to adjust for violations of the sphericity assumption. There were reliable main effects of both flanker duration $[F(5,75)=233.29, p<.001]$ and separation $[F(6,90)=44.23, p<.001]$. The interaction between flanker duration and separation was also significant $[F(30,450)=12.03, p<.001]$. Post hoc comparisons (Scheffé test, $\alpha=.05$ ) for the factor flanker duration confirmed that, moving from shortest to longest, each flanker duration yielded a significantly different visibility rating from the preceding one, except for the longest duration. Specifically, the central bar was rated as more and more invisible, the longer the flanker duration. The critical variable for the question addressed in this study was separation, and we found that although there was a main effect of separation, visibility ratings dropped rapidly with increasing flanker duration for all separations in a very similar way. Post hoc comparisons confirmed that the individual separations were very similar to each other: Separation 1 was not significantly different from Separation 2, Separation 2 was not different from Separation 3; Separation 3

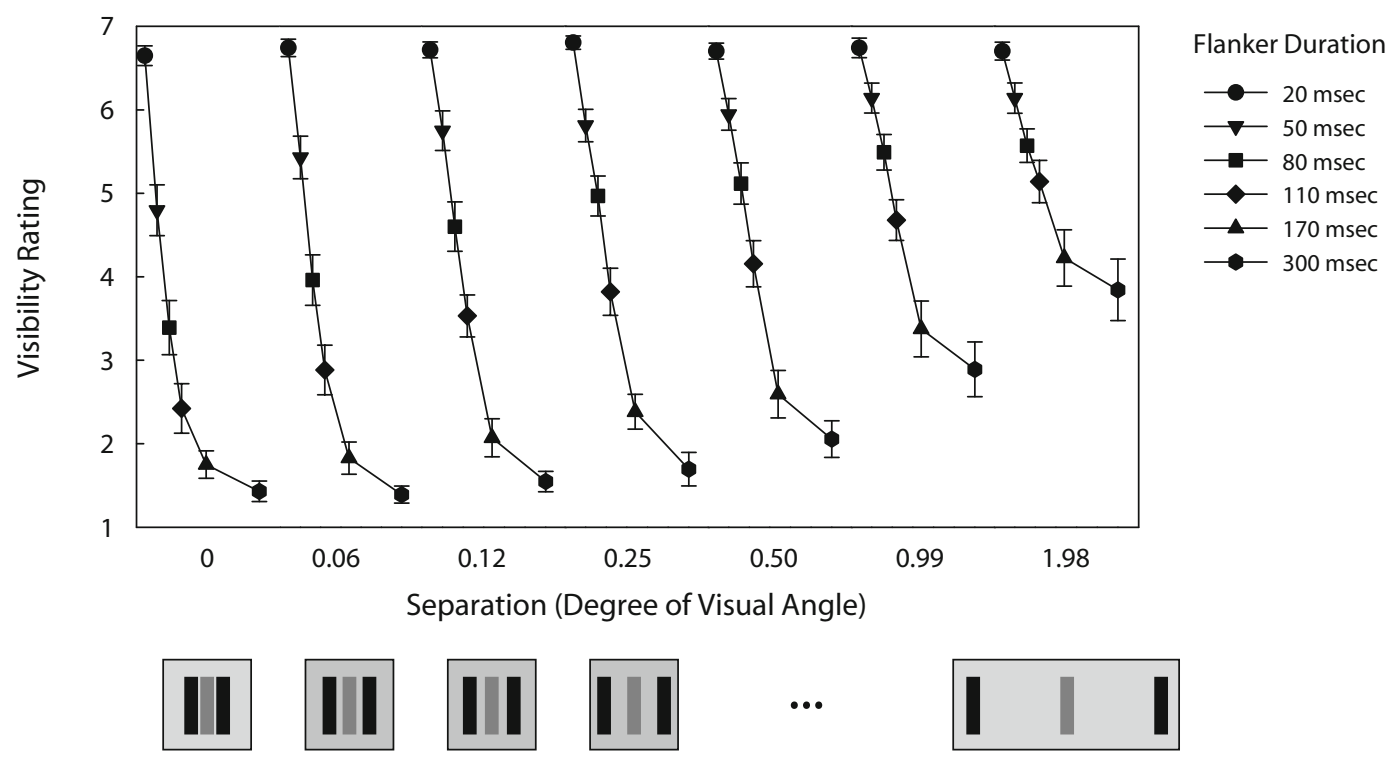

Figure 5. Mean visibility ratings on a scale of 1-7 $(1=$ invisible; $7=$ maximally visible $)$ as a function of flanker duration (in milliseconds) and separation (the horizontal distance between the sides of the central bar and the corresponding flankers) for Experiment 1. Error bars indicate the standard errors of the means. The central bar is rendered gray for illustration purposes only; in the experiment, it was presented at the same luminance level as the flankers. 
was not different from Separation 4 or 5; Separation 4 was not different from Separation 5; Separation 5 was not different from Separation 6; and finally, Separation 6 was not different from Separation 7.

The results of Experiment 1 show an effect of separation: Visibility of the central bar is increased with increasing distance between the central bar and the flankers. This suggests an influence of local mechanisms on the visibility of the central bar, as has been proposed in terms of low-level lateral inhibition (Macknik \& Livingstone, 1998; Macknik \& Martinez-Conde, 2004b) or ambiguous border ownership (Enns, 2002; Werner, 1935). Even though separation had an effect, visibility nonetheless dropped substantially with increasing flanker duration at all separations. Moreover, in all but the two most extreme separations, it dropped to below 3 , which means that even for a very clear separation of $0.5^{\circ}$, the central bar was judged as mostly invisible at the longest flanker durations. The flankers clearly did not share a border with the central bar at this separation, yet the central bar was rendered mostly invisible by the flanking bars. Therefore, explanations of the standing wave illusion in terms of local mechanisms (Enns, 2002; Macknik \& Livingstone, 1998; Werner, 1935) alone are insufficient to explain the entire pattern of results. The finding that visibility is reduced even with large separations between the central bar and the flankers suggests that object-level influences, as they were proposed by the object-updating hypothesis, also contribute to the phenomenon. ${ }^{1}$

\section{EXPERIMENT 2}

In this experiment, we eliminated the possibility of border ownership between the central and flanking bars by separating them vertically across frames (see Figure 3, right panel). In this case, the boundaries of the flankers and the central bar were never in close proximity, and there was no room for ambiguity of border ownership. The bars were placed to the left and right of fixation instead of above and below, to accommodate the manipulation of vertical separation. Otherwise, the experiment was similar in structure to Experiment 1. A low-level lateral inhibition account (Macknik \& Livingstone, 1998) and border competition accounts (Enns, 2002; Werner, 1935) predict that the central bar should be visible in all but the $0^{\circ}$-separation condition. In contrast, the object-updating account predicts that the visibility of the central bar will decrease with increasing flanker durations across all separations. This follows because, as long as the timing is right, updating should happen, resulting in the disappearance of the central bar and the flanking bars being seen to move upward and downward between their original position and the position of the correspondent central bar. As soon as the flanker duration gets too short (and separation too large) for object updating to happen, no motion should be seen anymore, and the flankers and the central bar should be seen flickering at their respective locations.

The participants were informed that the central bar could be displayed at various locations on a virtual vertical line between the flankers and that they should judge its visibility regardless of its vertical position.

\section{Results and Discussion}

Figure 6 shows the mean visibility ratings as a function of separation and flanker duration in Experiment 2. The observers again reported the central bar as highly visible at very short flanker durations $(20 \mathrm{msec})$ and nearly invisible at longer flanker durations $(170-300 \mathrm{msec})$. As in Experiment 1, this general pattern is evident at all of the separations. Also like in Experiment 1, the lowest visibility rating was not as low at the largest separations as it was at the closer separations.

Statistical analyses confirmed these apparent patterns. Mean visibility ratings for individual observers were submitted to a 7 (separations) $\times 6$ (flanker durations) ANOVA. There were again reliable main effects of both flanker duration $[F(5,75)=125.26, p<.001]$ and separation $[F(6,90)=28.67, p<.001]$, as well as a reliable interaction $[F(30,450)=2.19, p<.001]$. Post hoc comparisons (Scheffé test, $\alpha=.05$ ) for flanker duration confirmed that moving from shortest to longest, again each flanker duration yielded a significantly different visibility rating from the preceding one, except for the longest duration. Specifically, the central bar was rated as more and more invisible, the longer the flanker duration. Also as in Experiment 1, although there was a main effect of separation, visibility ratings dropped rapidly with increasing flanker duration for all separations in a very similar way. Post hoc comparisons confirmed that the patterns of the different separations were very similar; only the smallest and largest levels of separation differed significantly from each other.

Overall, the results and implications of Experiment 2 are very similar to those of Experiment 1. Separation had an effect on the visibility of the inner bar, but only for the larger separations, even though the separation was already substantial in the $0^{\circ}$-shift condition. Furthermore, visibility ratings overall were a little bit higher than those in Experiment 1 . This result again suggests that local mechanisms can influence visibility in the standing wave, as was found previously. But visibility nonetheless dropped substantially with increasing flanker duration at all separations. Moreover, in all but the two most extreme separations, the central bar was perceived as mostly invisible (visibility ratings dropped to below 3 ). In this experiment, even the closer separations were completely unambiguous with regard to border ownership and the proximity of borders, because they were all offset vertically. Thus, as in Experiment 1, these findings cannot be explained only in terms of ambiguous border ownership (Enns, 2002; Werner, 1935) or low-level lateral inhibition (Macknik \& Livingstone, 1998; Macknik \& Martinez-Conde, 2004b) and are consistent with the idea that object-based updating contributes to the effect.

\section{EXPERIMENT 3}

One implication of the object-updating account is that as the central bar and the flankers are seen as two instantiations of the same object, apparent motion should be seen between them (Lleras \& Moore, 2003), a possible explanation of the competing location information that 

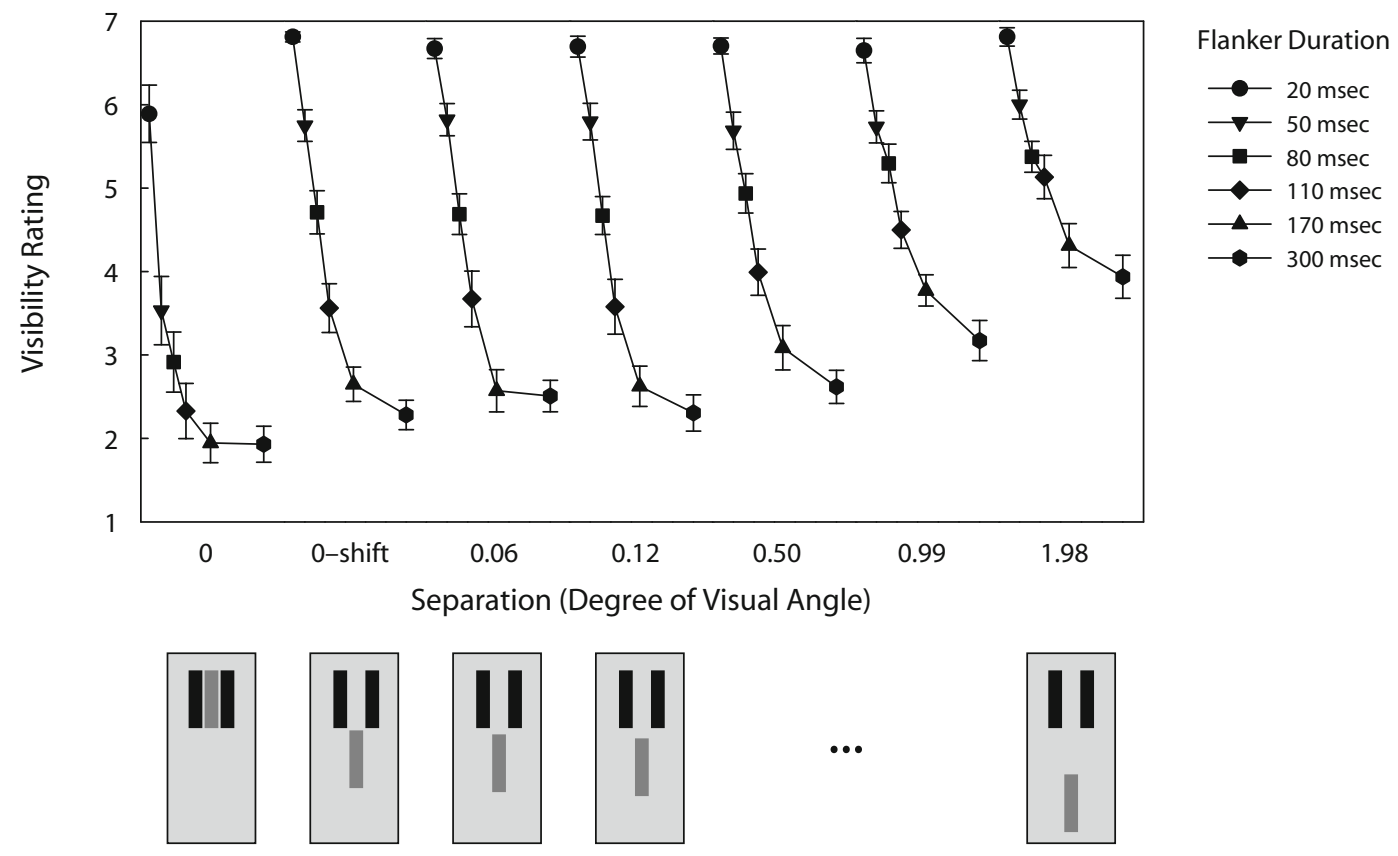

Figure 6. Mean visibility ratings on a scale of $1-7(1=$ invisible; $7=$ maximally visible $)$ as a function of flanker duration (in milliseconds) and separation (the vertical distance between the sides of the central bar and the corresponding flankers) for Experiment 2. "0" corresponds to the original standing wave display with no separation, " 0 -shift" corresponds to the first real separation, where the central bars were placed directly below (or above) the flankers' borders (cf. Figure 2). Error bars indicate the standard errors of the means. The central bar is rendered gray for illustration purposes only; in the experiment, it was presented at the same luminance level as the flankers.

has to be reconciliated in the unique object representation. In order to test this prediction, we asked the participants to report the quality of motion perception that they experienced between the central bar and the flankers (e.g., Kolers \& Pomerantz, 1971). Stimulus presentation was identical to that in Experiment 1. If the object-updating account is correct, good apparent motion should be perceived over the entire range of separations.

\section{Results and Discussion}

Figure 7 shows mean motion quality ratings as a function of separation and flanker duration in Experiment 3. As can be seen from the figure, the pattern is very similar to that of Experiment 1: Better motion was seen, the longer the flanker duration, and this was true for all of the separations tested.

Statistical analysis confirmed this pattern. Mean visibility ratings for individual observers were submitted to a 7 (separations) $\times 6$ (flanker durations) ANOVA. There were reliable main effects for flanker duration $[F(5,75)=281.89$, $p<.001]$ and for separation $[F(6,90)=3.05, p<.01]$, but unlike in Experiment 1, there was no significant interaction between these two factors $[F(30,450)=0.93$, n.s.]. Post hoc comparisons for the factor flanker duration confirmed that each duration was significantly different from all other durations, motion quality becoming better with increasing flanker duration. Furthermore, and even more interesting for our questions, post hoc comparisons for the factor separation revealed that the only significant difference was between the largest and the smallest separation.

Thus, the motion quality ratings were in line with the visibility ratings - the less the central bar was visible, the smoother the motion of the flankers was judged to beand this was true even for the largest separations. ${ }^{2}$ These findings are consistent with the object-updating account, suggesting that given the right timing, the central bar's representation becomes integrated with the representation of the flankers. As a consequence, the flankers are perceived as being in motion (moving between their physical position and the position of the central bar) in order to accommodate the fact that the stimuli occurred at multiple locations.

\section{GENERAL DISCUSSION}

The aim of this study was to test among different accounts of the standing wave illusion, which is a special form of lateral masking in which a central stimulus is cycled continuously with two flanking stimuli, rendering the central stimulus invisible. We suggest that the generation of the standing wave illusion is due to a combination of local mechanisms and object-updating mechanisms. According to this new object-updating view, the representation of the central stimulus is integrated with the representation of the flankers and therefore fails to be represented as a separate perceptual object, rendering it imperceptible as such. Traditional accounts - local lateral inhibition (e.g., Macknik 

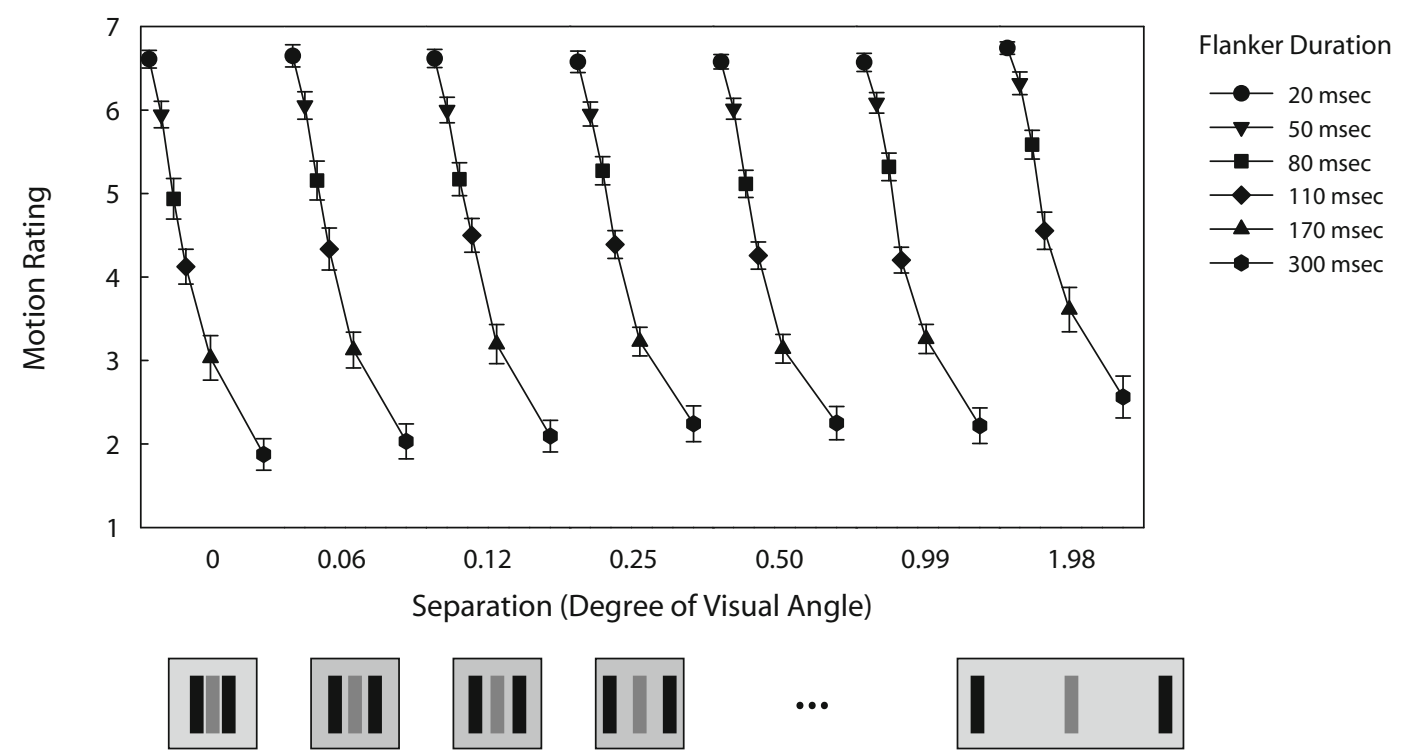

Figure 7. Mean motion quality ratings on a scale of $1-7(1=\operatorname{good}$ motion; $7=$ no motion $)$ as a function of flanker duration (in milliseconds) and separation (the horizontal distance between the sides of the central bar and the corresponding flankers) for Experiment 3. Error bars indicate the standard errors of the means. The central bar is rendered gray for illustration purposes only; in the experiment, it was presented at the same luminance level as the flankers.

\& Livingstone, 1998) and competition due to ambiguous border ownership (Enns, 2002; Werner, 1935)—attribute a critical role to the overlap, or very close proximity, of the outer border of the central bar and the inner boundaries of the flanking bars. The object-updating account does not. To test among these accounts, therefore, we conducted three experiments in which the central bar and its flankers were separated. Specifically, the flankers were displaced horizontally (Experiments 1 and 3) or vertically (Experiment 2). Consistent with both the local lateral inhibition and the border competition accounts, visibility of the central bar depended on separation. Consistent with the objectupdating account, however, visibility of the central bar was still substantially impaired even at separations as large as $1.98^{\circ}$ (Experiments 1 and 2). Moreover, and also consistent with the object-updating account, the participants rated the flankers as moving smoothly in conditions in which the central bar was reported as mostly invisible, whereas they tended to report seeing no motion in conditions in which the central bar was reported as visible (Experiment 3).

Although the entire pattern of results cannot be explained with a low-level lateral inhibition account of the standing wave illusion alone, it is consistent with an account in terms of high-level lateral inhibition circuits (Macknik, 2006; Macknik \& Martinez-Conde, 2004a, 2007). Large receptive-field sizes in later processing areas - for example, the inferotemporal cortex (IT) - would in principle allow for inhibitory competition across larger separations. Moreover, an inhibitory network in an area that is organized in terms of objects - rather than retinotopically, as is the IT (e.g., Kobatake \& Tanaka, 1994) — would provide an explanation for stronger inhibitory influences for similar than for dissimilar objects (Macknik \& Martinez-Conde,
2007). Thus, an inhibitory network within a high-level processing area could be a plausible neural instantiation of what we refer to here as an object-updating account. Such a high-level lateral inhibition account does not, however, explain why the reduced visibility of the central bar is accompanied by the perception of apparent motion. A lateral inhibitory circuit in the IT is therefore insufficient to capture the entire phenomenon.

Next, we offer a more detailed description of the objectupdating account of the standing wave illusion and relate it to other phenomena. It is assumed that when the visual system is faced with continuously changing information in the standing wave displays, it organizes the scene into a set of objects that are moving and changing over time. The idea is that the integration of newly sampled information into existing visual representations is mediated through the object representations that are formed through this organization process (e.g., Enns et al., 2008; Lleras \& Moore, 2003; Moore \& Lleras, 2005; Moore et al., 2007). To the extent that a stimulus is perceived as a later instantiation of an existing object within the current representation of the scene, that stimulus will be allowed to update that object representation. To the extent that a stimulus is perceived as a different represented object, or a new object, other object representations will be spared from updating on the basis of that stimulus. Under this view, the flanking bars in the standing wave situation are, under the right conditions, perceived as later instantiations of the central bar and, thus, integrate the representation of it, rendering it imperceptible as a separate entity. When the flanking bars are perceived as distinct objects, however, such as when the timing between frames is inappropriate to support apparent motion between the central bar and 
the flankers (or the central bar and the flankers are dissimilar), the information from the flanking stimuli is not integrated with that of the central bar, and the central bar is therefore visible.

A similar phenomenon was reported in the context of object substitution masking and was interpreted similarly by Lleras and Moore (2003). When a sparse mask, such as a set of four single-pixel dots arranged in a square pattern, is presented surrounding a target (e.g., a Landolt C) and remains for approximately $200 \mathrm{msec}$ or more following the offset of the target, the target can be rendered invisible (Di Lollo, Enns, \& Rensink, 2000; Enns \& Di Lollo, 1997). Lleras and Moore showed that the dots masked the target nearly as well when they offset along with the target but then appeared shortly afterward at a new location, thus giving rise to the perception of apparent motion. When the reappearance of the dot mask was delayed, however, so that it no longer supported good apparent motion, little or no masking occurred. Moreover, manipulations that were designed to encourage the organization of the target and mask as distinct objects (e.g., wiggling them independently of each other) decreased the amount of masking that occurred. In contrast, manipulations that were designed to encourage the organization of the target and mask as a single object (e.g., wiggling them together) increased the amount of masking that occurred (Moore \& Lleras, 2005). The explanation of these results in terms of objectmediated updating is that when the dots were perceived as a later instantiation of the target, such as when they were seen in apparent motion, the representation of the earlier stimulus became integrated with the information from the later stimulus and was thereby rendered inaccessible as a separate entity. This is clearly analogous to the account offered here of the standing wave illusion: The flankers mask the central bar because they are perceived as later instantiations of it.

The object-updating account suggests that the representation of the central bar is integrated into the representation of the flankers. A consequence of the account is therefore that the information associated with the central bar (i.e., its shape, surface attributes and location information) is not necessarily lost but is combined with the information collected about the flankers and thus changes the representation of the flankers. This is the reason that the flankers appear to be at two different locations over time: their own location and the location adopted by the central bar's original position. Moreover, this integration of representational information of the masked stimulus (i.e., the central bar) into the representation of the flankers could also happen for features other than location. Some observations by Werner (1935), as well as by Enns (2002), suggest that this may occur in the standing wave illusion. Werner, for example, reported that radiating spokes that are integrated in the central stimulus are sometimes perceived as belonging to the flanking stimulus instead. This phenomenon seems similar to that known as feature integration (e.g., Herzog \& Koch, 2001) in the masking literature.

Given this object-updating account, a natural question is why the flanking bars mask the central bar but not vice versa. The displays were continuously cycling. It is therefore not clear which stimulus should be interpreted as the later instantiation of which. Our data do not speak directly to this question, but we can speculate. A possible low-level account is that which stimulus dominatesflankers or central bar - may depend in part on the strength of the stimulus itself. The flanker duration varied in these experiments, whereas the central-bar duration did not. A consequence of this variation was that, integrating over time, the flankers were of higher intensity than the central bar, perhaps allowing the flankers to dominate perception. Only when the flanker duration was less than half as long as the central bar duration (a flanker duration of less than $50 \mathrm{msec}$ ) was the luminance of the flankers less than that of the central bar. In conditions in which this was the case, the central bar was visible, flickering at its central location, and no motion was perceived. Although plausible, it is unlikely that this explanation can fully account for the phenomenon. Informal observation shows that when there are only two bars cycling - one in the central position and one in one of the two flanker positions - the perception is of a single bar moving back and forth between those two positions, clearly visible at both positions. Thus, there seems to be something particular about there being a single bar cycling with two bars that renders the single bar invisible.

The correlation between reports of perceived motion and decreased visibility of the central bar (Experiment 3) suggests a strong relationship between masking and apparent motion. This is reminiscent of the debate regarding whether metacontrast masking and apparent motion reflect the same underlying mechanisms (e.g., Bischof \& Di Lollo, 1995; Breitmeyer \& Ganz, 1976; Di Lollo, Bischof, \& Dixon, 1993; Fehrer, 1966; Kahneman, 1967; Stoper \& Banffy, 1977). Although some researchers reported similarities between the two phenomena (e.g., Fehrer, 1966; Kahneman, 1967), others highlighted differences (e.g., Breitmeyer \& Horman, 1981; Kolers, 1972; Stoper \& Banffy, 1977; Weisstein \& Growney, 1969). Breitmeyer and Horman, for example, investigated the relationship between metacontrast masking and apparent motion by manipulating interstimulus interval $(0-225 \mathrm{msec})$ and separation $\left(0.14^{\circ}-3.21^{\circ}\right)$ between two stimuli and measuring both the magnitude of metacontrast masking and the quality of perceived motion. They found almost no difference in the reported quality of perceived motion across separations, but a strong dependence of metacontrast masking on separation. On the basis of this dissociation, they concluded that apparent motion is neither necessary nor sufficient for obtaining metacontrast masking.

The data from Experiments 1 and 2 of the present study may seem inconsistent with the findings of Breitmeyer and Horman (1981), in that we found substantial masking at large separations. The present study differed from Breitmeyer and Horman's, however, in that they used noncycling displays that included only two stimuli (i.e., the target and the mask), whereas we used cycling displays that included three stimuli (i.e., the central bar and two flanking bars). Again, informal observation indicates that when the central bar alternates with only one flanker, there is a greater balance between the visibility of the mask and 
that of the flanker. The critical difference seems to be the one-to-two relationship between stimuli across frames. The perceived motion is of an inward and outward oscillation from the location of the central bar, which is invisible, and the locations of the flankers, which are visible. We believe that this dependence on a one-to-two mapping and on the particular type of motion that is perceived (inward and outward) reflects the organization of the representation through which the updating of the cycling information is mediated.

Given the overall pattern, it seems likely that metacontrast masking and apparent motion are not identical but that they share a mechanism at some level (e.g., Kahneman, 1967). We suggest that object-mediated updating might be the underlying shared process. Depending on the similarity and timing between stimuli, the updating process can lead to both masking and apparent motion (see also Pilling \& Gellatly, 2009). In particular, both masking and apparent motion might occur through spatiotemporal integrative mechanisms (e.g., Reichardt, 1961), a consequence of which is that individual stimuli within a sequence of stimuli fail to be individuated in the perceptual representation (e.g., Bischof \& Di Lollo, 1995; Burr, 1984; Didner \& Sperling, 1980; Di Lollo et al., 1993; Fehrer, 1966; Schiller \& Smith, 1966). A study in which attention and scene organization are shown to modulate metacontrast masking suggests the same interpretation (Ramachandran \& Cobb, 1995; see also Werner, 1935).

Finally, other researchers have found a dependence of the magnitude of the standing wave illusion on the separation between the central bar and the flanker (Enns, 2002; Macknik, Martinez-Conde, \& Haglund, 2000; Werner, 1935). Enns, for example, included a manipulation of horizontal separation that was similar to the manipulation of separation used in Experiment 1 of the present study. The range of separations used in the Enns study ( 2 and 4 pixels, or $0.07^{\circ}$ and $0.13^{\circ}$ ), however, was much smaller than that used in the present study $\left(0.06^{\circ}-1.98^{\circ}\right)$. In fact, Enns found a reduction in the effect of the flanking bars with separation that was similar to the small reduction found in the present study across the small end of the range of separations. However, the standing wave illusion was far from eliminated. Moreover, when we extended the range out to larger separations, where it was unlikely that there would be any ambiguity of border ownership, there was still a large reduction in the visibility of the central bar because of the flankers. Lateral inhibition (Macknik \& Livingstone, 1998; Macknik et al., 2000) and/or competition due to ambiguous border ownership (Enns, 2002) may well contribute to the invisibility of the central bar when they are near each other. The effect, however, does not depend entirely on these local accounts. If it did, it would be eliminated at large separations.

Macknik et al. (2000) also reported a separation effect. In the first part of their study, they recorded the responses of single cells in monkey LGN in the standing wave display and found that with increasing distance between a central bar and flankers, the firing pattern related to the cycling presentation of a central bar and flankers corresponds more and more to the firing pattern of the presentation of the central bar alone. Although this study shows a dependence of separation, it is unclear how this result is related to perceived visibility and motion ratings.

In the second part of Macknik et al.'s (2000) study, a human psychophysics experiment, they used noncycling meta- and paracontrast masking displays, in which a central bar target was surrounded by a rectangle of various sizes, such that larger rectangles were separated from the central bar more than smaller rectangles were. Consistent with the metacontrast literature, they found that with increasing border separation, the central bar became more and more visible. It is difficult to compare this separation manipulation with those in the present study and that of Enns (2002), in part because they were noncycling. In addition, as the rectangles became bigger, they also became more dissimilar from the target. Thus, the effect of separation may reflect an effect of shape similarity, like those reported by Werner (1935) and Enns. As the central bar and mask become more dissimilar, they are less likely to be perceived as different instantiations of one object. As a consequence, the representation of the central bar is more likely to be protected from being overwritten by the surrounding rectangles (see also Moore \& Enns, 2004; Moore et al., 2007).

In summary, the present results cannot be explained only by accounts of the standing wave illusion that depend on the overlap or close proximity of the outer and inner boundaries of the central and flanking stimuli (Enns, 2002; Macknik \& Livingstone, 1998; Werner, 1935). We suggest that a representational updating process that is mediated through object representations contributes to the reduced visibility of the central bar. This account is consistent with the invisibility of the central bar in the standing wave illusion across large separations but also with the perception of motion that is experienced during these displays. It is also consistent with a variety of related phenomena, including the influence of shape similarity between the central bar and the flankers (Enns, 2002; Werner, 1935), the impact of perceptual organization on masking (Ramachandran \& Cobb, 1995; Werner, 1935), and the migration of features between the central bar and the flankers, such as gray level, size, and texture (Enns, 2002; Werner, 1935).

\section{AUTHOR NOTE}

This work has been supported by NSF Grant BCS-0818536 to C.M.M. We thank Stefan Blaschke and John Palmer for helpful discussions, as well as Haluk Öğmen, Jim Enns, and two anonymous reviewers for valuable comments on previous versions of the manuscript. Correspondence concerning this article should be addressed to E. Hein, Department of Psychology, E11 Seashore Hall, University of Iowa, Iowa City, IA 52242 (e-mail: elisabeth-hein@uiowa.edu).

\section{REFERENCES}

Bischof, W. F., \& Di Lollo, V. (1995). Motion and metacontrast with simultaneous onset of stimuli. Journal of the Optical Society of America A, 12, 1623-1636.

Brainard, D. H. (1997). The Psychophysics Toolbox. Spatial Vision, 10, 433-436.

Breitmeyer, B. G., \& GANZ, L. (1976). Implications of sustained and 
transient channels for theories of visual pattern masking, saccadic suppression, and information processing. Psychological Review, 83, $1-36$.

Breitmeyer, B. G., \& Horman, K. (1981). On the role of stroboscopic motion in metacontrast. Bulletin of the Psychonomic Society, 17, 29-32.

Breitmeyer, B. G., \& ÖĞMen, H. (2000). Recent models and findings in visual backward masking: A comparison, review, and update. Perception \& Psychophysics, 62, 1572-1595.

Breitmeyer, B. G., \& ÖĞMEN, H. (2006). Visual masking: Time slices through conscious and unconscious vision. Oxford: Oxford University Press.

BURR, D. C. (1984). Summation of target and mask metacontrast stimuli. Perception, 13, 183-192.

DiDner, R., \& SPERling, G. (1980). Perceptual delay: A consequence of metacontrast and apparent motion. Journal of Experimental Psychology: Human Perception \& Performance, 6, 235-243.

Di Lollo, V., Bischof, W. F., \& Dixon, P. (1993). Stimulus onset asynchrony is not necessary for motion perception or metacontrast masking. Psychological Science, 4, 260-263.

Di Lollo, V., EnNS, J. T., \& Rensink, R. A. (2000). Competition for consciousness among visual events: The psychophysics of reentrant visual processes. Journal of Experimental Psychology: General, 129, 481-507.

Duangudom, V., Francis, G., \& Herzog, M. H. (2007). What is the strength of a mask in visual metacontrast masking? Journal of Vision, 7(1, Art. 7), 1-10. doi:10.1167/7.1.7

EnNs, J. T. (2002). Visual binding in the standing wave illusion. Psychonomic Bulletin \& Review, 9, 489-496.

EnNs, J. T., \& Di Lollo, V. (1997). Object substitution: A new form of masking in unattended visual locations. Psychological Science, 8, 135-139.

Enns, J. T., Lleras, A., \& Moore, C. M. (2008). The contribution of object updating to perceptual continuity and scene stability in human vision. In R. Nihjawan (Ed.), Issues of space and time in perception and action. Cambridge: Cambridge University Press.

FeHrer, E. (1966). Effect of stimulus similarity on retroactive masking. Journal of Experimental Psychology, 71, 612-615.

FranCIS, G. (1997). Cortical dynamics of lateral inhibition: Metacontrast masking. Psychological Review, 104, 572-594.

Herzog, M. H., Fahle, M., \& Koch, C. (2001). Spatial aspects of object formation revealed by a new illusion, shine-through. Vision Research, 41, 2325-2335.

Herzog, M. H., \& Koch, C. (2001). Seeing properties of an invisible object: Feature inheritance and shine-through. Proceedings of the $\mathrm{Na}$ tional Academy of Sciences, 98, 4271-4275.

KAHNEMAN, D. (1967). An onset-onset law for one case of apparent motion and metacontrast. Perception \& Psychophysics, 2, 577-584.

KobataKe, E., \& TanaKa, K. (1994). Neuronal selectivities to complex object features in the ventral visual pathway of the macaque cerebral cortex. Journal of Neurophysiology, 71, 856-867.

Kolers, P. A. (1972). Aspects of motion perception. New York: Pergamon.

Kolers, P. A., \& Pomerantz, J. R. (1971). Figural change in apparent motion. Journal of Experimental Psychology, 87, 99-108.

KorTe, A. (1915). Kinematoskopische Untersuchungen. Zeitschrift für Psychologie, 72, 194-296.

Lleras, A., \& Moore, C. M. (2003). When the target becomes the mask: Using apparent motion to isolate the object-level component of object substitution masking. Journal of Experimental Psychology: Human Perception \& Performance, 29, 106-120.

MACKNIK, S. L. (2006). Visual masking approaches to visual awareness. Progress in Brain Research, 155, 177-215.

MaCKNiK, S. L., \& Livingstone, M. S. (1998). Neuronal correlates of visibility and invisibility in the primate visual system. Nature Neuroscience, 1, 144-149.

Macknik, S. L., \& Martinez-Conde, S. (2004a). Dichoptic visual masking reveals that early binocular neurons exhibit weak interocular suppression: Implications for binocular vision and visual awareness. Journal of Cognitive Neuroscience, 16, 1049-1059.

Macknik, S. L., \& Martinez-Conde, S. (2004b). The spatial and temporal effects of lateral inhibitory networks and their relevance to the visibility of spatiotemporal edges. Neurocomputing, 58-60, 775-782.

MaCKNiK, S. L., \& MARTINEZ-Conde, S. (2007). The role of feedback in visual masking and visual processing. Advances in Cognitive Psychology, 3, 125-152.

Macknik, S. L., Martinez-Conde, S., \& Haglund, M. M. (2000) The role of spatiotemporal edges in visibility and visual masking. Proceedings of the National Academy of Sciences, 97, 7556-7560.

Moore, C. M., \& EnNS, J. T. (2004). Substitution masking and the flashlag effect. Psychological Science, 15, 866-871.

Moore, C. M., \& Lleras, A. (2005). On the role of object representations in substitution masking. Journal of Experimental Psychology: Human Perception \& Performance, 31, 1171-1180.

Moore, C. M., Mordkoff, J. T., \& EnNs, J. T. (2007). The path of least persistence: Object status mediates visual updating. Vision Research, 47, 1624-1630.

Pelli, D. G. (1997). The VideoToolbox software for visual psychophysics: Transforming numbers into movies. Spatial Vision, 10, 437-442.

Pilling, M., \& Gellatly, A. (2009). Target visibility in the standing wave illusion: Is mask-target shape similarity important? Perception, 38, 5-16.

RAMACHANDRAN, V. S., \& CoвB, S. (1995). Visual attention modulates metacontrast masking. Nature, 373, 66-68.

REICHARDT, W. (1961). Autocorrelation, a principle for the evaluation of sensory information by the central nervous system. In W. A. Rosenblith (Ed.), Sensory communication (pp. 303-317). Cambridge, MA: MIT Press.

Schiller, P. H., \& SMith, M. C. (1966). Detection in metacontrast. Journal of Experimental Psychology, 71, 32-39.

Stoper, A. E., \& BANFFy, S. (1977). Relation of split apparent motion to metacontrast. Journal of Experimental Psychology: Human Perception \& Performance, 3, 258-277.

Weisstein, N. (1972). Metacontrast. In D. Jameson \& L. M. Hurvich (Eds.), Handbook of sensory physiology (Vol. III/4, pp. 233-272). New York: Springer-Verlag.

Weisstein, N., \& Growney, R. L. (1969). Apparent movement and metacontrast: A note on Kahneman's formulation. Perception \& Psychophysics, 5, 321-328.

Werner, H. (1935). Studies of contour: I. Qualitative analyses. American Journal of Psychology, 47, 40-64.

\section{NOTES}

1. Theoretically, the object-updating account could also explain the dependence of visibility on separation. As separation becomes very large, the interpretation of the scene becomes more and more ambiguous. Instead of individual bars moving in a very short time over a large distance, the bars are more and more likely to sometimes be seen as stationary bars flashing at their position, each single bar becoming perfectly visible. This situation would increase the variability in responses, as it is observed in our data.

2. We conducted a pilot experiment with 4 naive participants, obtaining motion ratings for the displays used in Experiment 2. The same pattern of results was observed.

\section{SUPPLEMENTAL MATERIALS}

Eight QuickTime movie files illustrating the key conditions of our experiments may be downloaded from http://app.psychonomic-journals .org/content/supplemental.

(Manuscript received January 14, 2009; revision accepted for publication October 9, 2009.) 